\title{
Strain improvement of Rhizopus oryzae for over- production of lactic acid by random mutations
}

\author{
Longfei Yin, Qicheng Ruan and Yongqian Fu* \\ Institute of Biomass Resources, Taizhou University, Taizhou 317300, China. \\ Accepted 29 March, 2013
}

\begin{abstract}
Rhizopus oryzae mutant strain LA-UN-1 was isolated from the lactic acid-producing strain NRRL 395 by mutagenesis with both ultraviolet (UV) irradiation and nitrosoguanidine (NTG) treatment with the aim of selecting mutant in pellet form. One of the mutants in flask culture easily formed the pellet morphology at a diameter between 1.1 and $1.3 \mathrm{~mm}$. In this case, the lactic acid concentration reached $59.5 \mathrm{~g} / \mathrm{l}$, which was $54.5 \%$ higher than that of the wild type. This investigation indicates that the production of L-lactic acid could be enhanced with the method of mutagenesis.
\end{abstract}

Key words: Lactic acid, morphology, mutagenesis, Rhizopus oryzae.

\section{INTRODUCTION}

$\mathrm{L}-(+)$-lactic acid is a natural organic acid that is widely applied in food, pharmaceutical, cosmetics and chemical industries. It can also be used in the production of polylactic acid, a precursor to biodegradable plastic. Lactic acid can be produced using bacteria and fungi. Rhizopus oryzae NRRL 395 is known to have strong ability for producing optically pure lactic acid (Oda et al., 2002; Liu et al., 2006; Meussen et al., 2012; Liu et al., 2005). A great deal of research about the production of lactic acid by Rhizopus fungi including process engineering, metabolic and enzymatic mechanisms, and molecular biotechnology have been carried out (Zhang et al., 2007).

As filamentous mycelia, Rhizopus species can grow in different morphological forms such as clumps, filaments, and pellets (Kossen, 2000). The particular form of morphology is determined not only by the genetic material of the fungal species but also the nature of inoculum as well as the chemical (medium constituents) and physical $(\mathrm{pH}$, temperature, mechanical forces, spore concentration) culturing conditions (Kossen, 2000; Büyükkileci et al., 2006). The morphology of filamentous fungi during fermentation processes is critical to maximum performance. Free filamentous mycelia not only lead to a highly viscous fermentation broth that reduces the gas-liquid mass transfer and the homogeneity in reactors, but also may block aeration nozzles, cling to impellers and baffles, and form large cakes (Park et al., 1998). The pellet form can overcome these problems, so pellets are often the preferable morphological forms in industrial fermentation processes. Many studies have also demonstrated that it can improve the yield of product with Rhizopus species by controlling the pelleted morphology (Byrne and Ward, 1989; Žnidaršič et al., 2000; Zhou et al., 2000; ŽnidaršičPlazl, 2006).

Production of lactic acid with pelleted $R$. oryzae NRRL 395 was also enhanced in a stirred fermentor using fedbatch culture (Liu et al., 2006). Suitable pellet morphology for the production of lactic acid by Rhizopus species could also be formed (Miura et al., 2003; Du et al., 1998; Yin et al., 1998). However, these models and practices were based almost on the influence of environmental factors on morphology. Because environmental factors vary considerably during production phase as well as on scale, this could make morphogenesis rather difficult to control. What is more, although the genome of $R$. oryzae strain 99-880 was published (Ma et al., 2009), the molecular mechanism behind morphogenesis has not been very well elucidated until now and genetically controlled morphogenesis has not been realized. Strain improvement has been conventionally achieved through mutation 

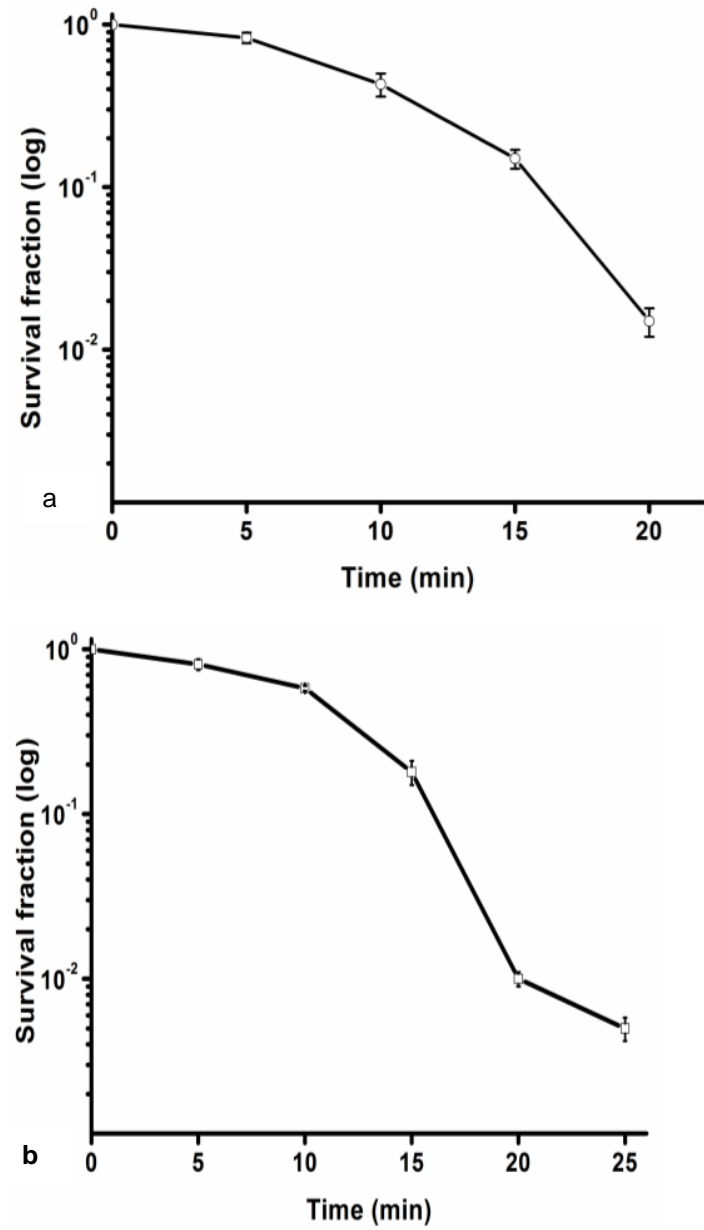

Figure 1. Survival curves of Rhizopus oryzae NRRL 395 after (a) UV irradiation and (b) treatment of NTG. After spores were treatment, $0.25 \mathrm{ml}$ spores suspension was spread onto the potato-dextrose agar plates at $30^{\circ} \mathrm{C}$ for 3 days. Survival rate was expressed as the number of colonies obtained from treated samples as a percentage of the number for untreated controls. Values are means \pm $\mathrm{SD}$ of three independent experiments.

and selection. So based on the importance of morphology and the current knowledge, in this investigation, the mutagenesis of the wild type strain, $R$. oryzae NRRL 395, with ultraviolet (UV) coupled with nitrosoguanidine (NTG) treatment was conducted by selecting the pelleted morphology strain in flask culture. And the production of lactic acid was also performed in a stirred bioreactor. To our knowledge, it is the first report that strain improvement of $R$. oryzae for production of organic acid by mutagenesis was based on morphology changed.

\section{MATERIALS AND METHODS}

\section{Microorganism and culture media}

R. oryzae NRRL 395 (Lab stock) was used as the initial strain in this study. The seed medium ( $\mathrm{g} / \mathrm{l}$ ) contained soluble starch 10, pep- tone 5.0, yeast extract 5.0, $\mathrm{KH}_{2} \mathrm{PO}_{4} 0.2$ and $\mathrm{MgSO}_{4} \cdot 7 \mathrm{H}_{2} \mathrm{O}$ 0.2. The fermentation medium $(\mathrm{g} / \mathrm{l})$ consisted of glucose $90, \mathrm{NH}_{4} \mathrm{SO}_{4} 3.02$, $\mathrm{MgSO}_{4} \cdot 7 \mathrm{H}_{2} \mathrm{O} \quad 0.25, \mathrm{KH}_{2} \mathrm{PO}_{4}, 0.15$ and $\mathrm{ZnSO}_{4} \cdot 7 \mathrm{H}_{2} \mathrm{O}$ 0.04. The media were autoclaved at $121^{\circ} \mathrm{C}$ for $20 \mathrm{~min}$.

\section{Culture conditions}

The fungus was grown on potato-dextrose agar slants at $30^{\circ} \mathrm{C}$ for 6 days. For the experiments, the fungal spores in the slants were collected with an inoculating loop and suspended in sterilized water maintained at $4^{\circ} \mathrm{C}$. The spore solution was inoculated into a $250 \mathrm{ml}$ Erlenmeyer flask containing $50 \mathrm{ml}$ seed medium with a spore concentration of $10^{6}$ spores $/ \mathrm{ml}$. The flasks were placed on a rotary shaker at $30^{\circ} \mathrm{C}$ and $170 \mathrm{rpm}$ for $24 \mathrm{~h}$.

The production of lactic acid was performed in a $3 \mathrm{~L}$ stirred bioreactor (NBS, New Brunswick, USA) containing 2 L fermentation medium which was inoculated with $300 \mathrm{ml}$ seed culture. The culture temperature in the bioreactor was maintained at $30^{\circ} \mathrm{C}$ throughout the experiment. The aeration rate and agitation speed were set at 1.0 and $200 \mathrm{rpm} / \mathrm{min}$, respectively. The $\mathrm{pH}$ was adjusted at 6.0 with sterile $\mathrm{CaCO}_{3}$.

\section{Mutagenesis and morphology detection in flask culture}

The experiments for mutagenizing the wild type strain with UV irradiation coupled with NTG treatment were performed mainly according to the previous methods (Suntornsuk and Hang, 1994). Firstly, the spore suspension $\left(10^{8}\right.$ spores $\left./ \mathrm{ml}\right)$ was irradiated with a $30 \mathrm{~W}$ UV lamp at $254 \mathrm{~nm}$ at a distance of $20 \mathrm{~cm}$. Then the spore suspension was treated with $100 \mathrm{~g} / \mathrm{ml} \mathrm{NTG}$ in phosphate buffer $(\mathrm{pH}$ 7.0) at $30^{\circ} \mathrm{C}$. Finally, spores suspension was spread onto the potato-dextrose agar plates at $30^{\circ} \mathrm{C}$ for 3 days. The single colony on plates was selected to be cultured in the seed medium. The morphology of $R$. oryzae mutants in flask culture was observed. The pelleted morphology of $R$. oryzae was chosen for further study. The survival rates of $R$. oryzae NRRL 395 after UV irradiation or treatment of $100 \mathrm{~g} / \mathrm{ml} \mathrm{NTG}$ for different time were determined.

\section{Analyses of metabolic products}

For glucose concentration determination, samples were collected by centrifugation and the supernatants were used. For lactic acid concentration determination, samples were diluted by distilled water and heated at $80^{\circ} \mathrm{C}$ until the culture liquid was clear. Then the diluted suspensions were centrifuged and the supernatants were used. The concentration of glucose and lactic acid were measured by a biosensor (Institute of Biology, Shangdong Academy of Sciences SBA-40D) with glucose oxidase and lactate oxidase electrode, respectively.

\section{RESULTS AND DISCCUSION}

\section{Determination of survival rate}

To determine the treatment time, the survival rate of $R$. oryzae NRRL 395 was detected. As shown in Figure 1, the spore survival of $R$. oryzae NRRL 395 after treatment with UV irradiation or NTG was reduced gradually by increasing the period of exposure to mutagenic agents. This result was almost compatible with the previous result (Suntornsuk and Hang, 1994). In this study, a combined treatment based on the survival UV irradiation for $10 \mathrm{~min}$ 

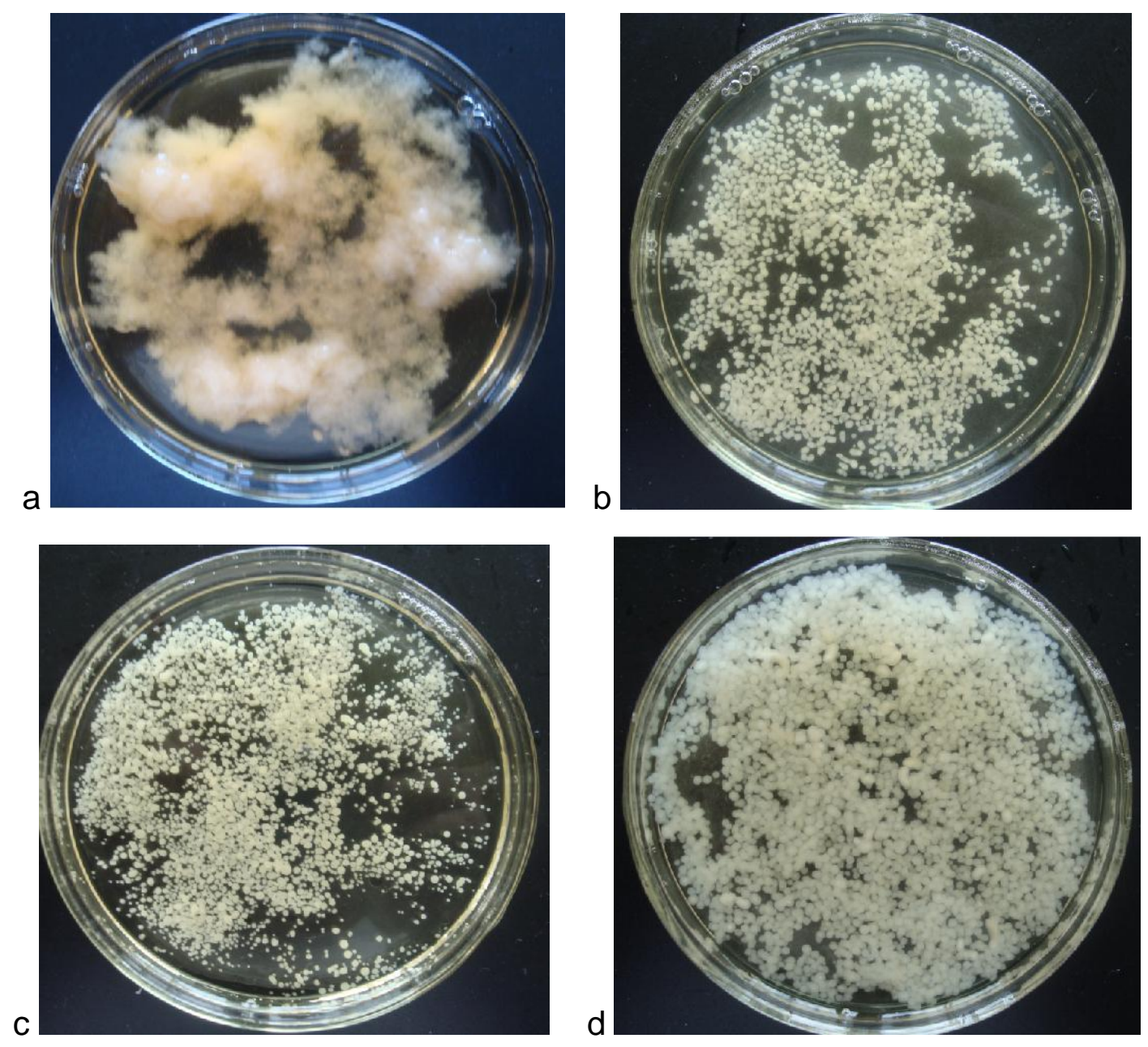

Figure 2. The morphology of Rhizopus oryzae NRRL 395 and mutants in $250 \mathrm{ml}$ flask culture at the same culture condition. The photos were taken from a $12 \mathrm{~cm}$ Petri-dish at the end of the cultivations by digital camera. (a) Rhizopus oryzae NRRL 395, (b) LA-UN-1, (c) LA-UN-2, (d) LA-UN-3.

followed by treatment of NTG for $10 \mathrm{~min}$. The survival rate after the combined treatment was rate was performed with $11 \%$.

\section{Morphology observation of mutants}

After mutagenesis, several morphological mutants were successfully obtained. As shown in Figure 2, mutants LAUN-1, 2, 3 formed pelleted morphology in flask culture and NRRL 395 showed filamentous morphology at the same cultivation condition. But the diameters of pelleted morphology among mutants in flask culture were different. The diameter of mutant LA-UN-1 pellet was between 1.1 and $1.3 \mathrm{~mm}$. The diameter of mutants LA-UN-2 and LA-UN- 3 were $0.6-0.8$ and $1.4-1.6 \mathrm{~mm}$, respectively. Although mycelia pellet are often the preferable morphological forms in industrial fermentation processes, different size of the pellets can pose problems about the transfer of nutrients and oxygen to the interior of the pellet. So to compare the ability of producing lactic acid, the fermentation of mutants were performed. LA-UN-1 showed stronger ability for producing lactic acid than LAUN-2, 3 (Figure 3) and the morphology of LA-UN-1 in bioreactor was shown in the Figure $4 \mathrm{a}$. This demonstrates that about $1.2 \mathrm{~mm}$ of the diameter of pellet may be a better size for production of lactic acid. It was ever reported that the critical pellet size of $R$. oryzae for producing fumaric acid was also $1.2 \mathrm{~mm}$ (Fu et al., 2009).

Although pellets of $R$. oryzae inoculated in shake flasks are also formed by controlling the culture conditions such as inoculum size, heavy metal and nitrogen concentrations (Zhou et al., 2000; Liao et al., 2007), it was still interesting that the pelleted morphology was formed by UV irradiation and NTG treatment. Alteration of gene related to the morphology formation may be involved in the treatment in this investigation. Until now the mechanism about the formation of pelleted morphology has not been interpreted. The molecular mechanism about morphological change of $R$. oryzae after mutagenesis is being 


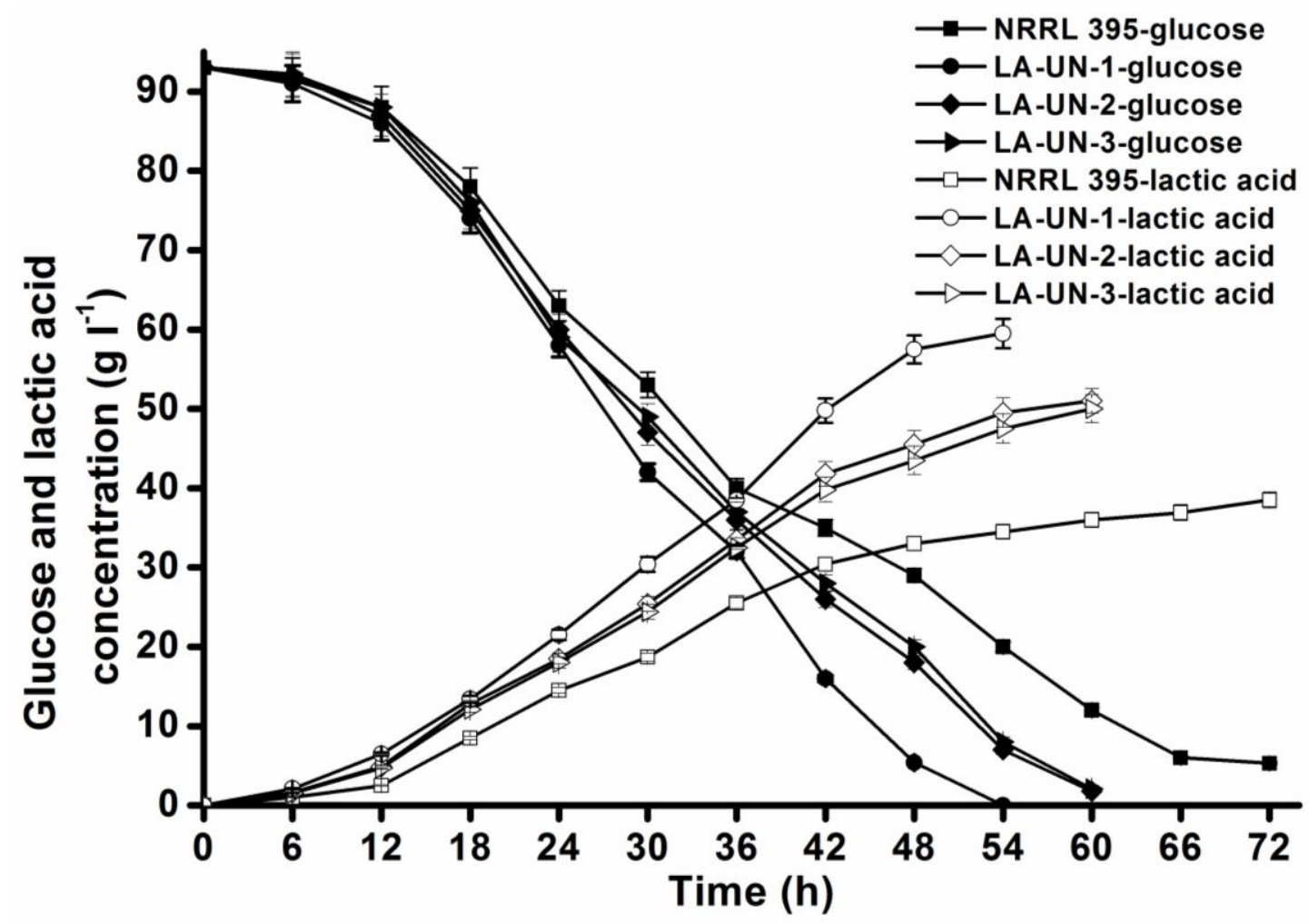

Figure 3. Time-course of L-lactic acid production by Rhizopus oryzae NRRL 395 and mutant strains LA$\mathrm{UN}-1,2,3$ from glucose in $3 \mathrm{~L}$ stirred bioreactor.
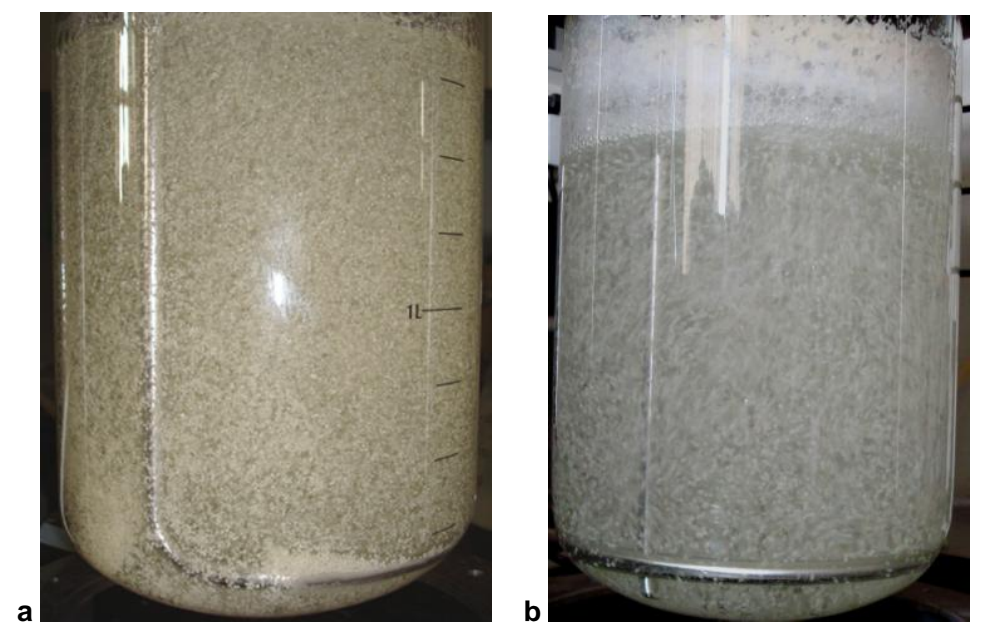

Figure 4. The morphology of LA-UN-1 in bioreactor (a) and after the 10th run of the repeated batch culture (b).

investigated in our laboratory by molecular biotechnology.

\section{Analysis of lactic acid production in stirred bioreactor}

Lactic acid fermentation with NRRL 395 and mutant LAUN-1, 2, 3 were carried out in bioreactor (Figure 3). The results indicate that there was significant difference in lactic acid production among the strains. The lactic acid concentration of NRRL 395 was $38.5 \mathrm{~g} / \mathrm{l}$ in $72 \mathrm{~h}$ of fermentation duration whereas the one by the mutant LA$\mathrm{UN}-1$ reached to $59.5 \mathrm{~g} / \mathrm{l}$ after54 $\mathrm{h}$. The lactic acid production of LA-UN-1 was $54.5 \%$ more than the wild type. The lactic acid yield of LA-UN-2, 3 were also much higher 
Table 1. Concentration and yield of lactic acid after repetitive batch fermentation with LA-UN-1 in bioreactor. Values are means \pm SD of three independent experiments.

\begin{tabular}{lcc}
\hline Batch number & Concentration $(\mathbf{g} / \mathbf{l})$ & Yield $\left.^{(\%)}\right)^{\mathbf{a}}$ \\
\hline 1 & $59.5 \pm 1.37$ & $63.98 \pm 1.47 \%$ \\
2 & $58.7 \pm 1.17$ & $63.12 \pm 1.26 \%$ \\
3 & $59.1 \pm 1.47$ & $63.55 \pm 1.58 \%$ \\
4 & $58.5 \pm 1.75$ & $62.90 \pm 1.88 \%$ \\
5 & $59.4 \pm 1.90$ & $63.87 \pm 2.04 \%$ \\
6 & $60.5 \pm 1.87$ & $65.05 \pm 2.01 \%$ \\
7 & $59.1 \pm 1.24$ & $63.55 \pm 1.33 \%$ \\
8 & $60.1 \pm 1.38$ & $64.62 \pm 1.48 \%$ \\
9 & $58.3 \pm 1.34$ & $62.69 \pm 1.44 \%$ \\
10 & $59.5 \pm 1.01$ & $63.98 \pm 1.08 \%$ \\
\hline
\end{tabular}

${ }^{a}$ Yield $(\%)=$ gram of lactic acid produced per gram of total glucose consumed.

than that of the wild type. These data demonstrate that the pelleted morphology of $R$. oryzae could improve the lactic acid production. Liu et al. (2006) also proved that pelleted $R$. oryzae has a stronger ability for pro-ducing lactic acid than filamentous one. Fu et al. (2009) reported that $R$. oryzae could enhance the fumaric acid production by controlling the pelleted morphology. Figure 3 also indicates that LA-UN-1 costumed glucose in much shorter period than wild type during producing lactic acid.

\section{Repeated batch culture in bioreactor with LA-UN-1}

Repetitive batch fermentation was carried out in bioreactor in order to demonstrate the stability of the LA-UN1 (Table 1). As shown in Table 1, the produced lactic acid and the yield of L-lactic acid based on the consumed glucose were above $58.3 \mathrm{~g} / \mathrm{l}$ and $52.69 \%$ in 10 batches of repetitive fermentation, respectively. This result shows that the mutant LA-UN-1 has high stability for producing lactic acid. At the same time, the morphology of the strain after 10 batches fermentation was still pelleted (Figure $4 b)$. These results above demonstrate that LA-UN-1 screened in this investigation seems to be a promising strain for further lactic acid production, and the mutagenesis and selection method developed in this study are useful to screen the high-lactic acid-producing strain.

\section{ACKNOWLEDGMENT}

This work was supported by a grant from the National Natural Science Foundation of China (21106091) and a grant from Zhejiang Provincial Natural Science Foundation of China (No. LQ12B06004).

\section{REFERENCES}

Büyükkileci AO, Hamamci $H$, Yucel M (2006). Lactate and ethanol productions by Rhizopus oryzae ATCC 9363 and activities of related pyruvate branch point enzymes. J. Biosci. Bioeng. 102: 464-466..
Byrne GS and Ward OP (1989). Effect of nutrition on pellet formation by Rhizopus arrhizus. Biotechnol. Bioeng. 33: 912-914.

Du J, Cao N, Gong CS, Tsao GT (1998). Production of L-lactic acid by Rhizopus oryzae in a bubble column fermenter. Appl. Biochem.

Biotechnol. 70-72: 323-329.

Fu Y, Xu Q, Li S, Huang $H$, Chen $Y$ (2009). A novel multi-stage preculture strategy of Rhizopus oryzae ME-F12 for fumaric acid production in a stirred-tank reactor. World J. Microbiol. Biotechnol. 25: $1871-1876$.

Kossen NW (2000). The morphology of filamentous fungi. Adv. Biochem. Eng. Biotechnol. 70: 1-33.

Liao W, Liu Y, Chen S (2007). Studying pellet formation of a filamentous fungus Rhizopus oryzae to enhance organic acid production. Appl. Biochem. Biotechnol. 137-140: 689-701.

Liu Y, Liao W, Liu C, Chen S (2006). Optimization of L-(+)-lactic acid production using pelletized filamentous Rhizopus oryzae NRRL 395. Appl. Biochem. Biotechnol. 131: 844-853.

Liu Y, Wen Z, Liao W, Liu C, Chen S (2005). Optimization of the process for the production of $L(+)$-actic acid from cull potato by Rhizopus oryzae. Eng. Life Sci. 5: 343-349.

Ma LJ, Ibrahim AS, Skory C, Grabherr MG, Burger G, Butler M, Elias M, Idnurm A, Lang BF, Sone T, Abe A, Calvo SE, Corrochano LM, Engels R, Fu J, Hansberg W, Kim JM, Kodira CD, Koehrsen MJ, Liu B, Miranda-Saavedra D, O'Leary S, Ortiz-Castellanos L, Poulter R, Rodriguez-Romero J, Ruiz-Herrera J, Shen YQ, Zeng Q, Galagan J, Birren BW, Cuomo CA, Wickes BL (2009). Genomic analysis of the basal lineage fungus Rhizopus oryzae reveals a whole-genome duplication. PLoS Genet. 5: e1000549.

Meussen BJ, de Graaff LH, Sanders JP, Weusthuis RA (2012). Metabolic engineering of Rhizopus oryzae for the production of platform chemicals. Appl. Microbiol. Biotechnol. 94: 875-886.

Miura S, Arimura T, Hoshino M, Kojima M, Dwiarti L, Okabe M (2003). Optimization and scale-up of L-lactic acid fermentation by mutant strain Rhizopus sp. MK-96-1196 in airlift bioreactors. J. Biosci. Bioeng. 96: 65-69.

Oda Y, Saito K, Yamauchi H, Mori M (2002). Lactic acid fermentation of potato pulp by the fungus Rhizopus oryzae. Curr. Microbiol. 45: 1-4.

Park EY, Kosakai Y, Okabe M (1998). Efficient production of L-(+)-lactic acid using mycelial cotton-like flocs of Rhizopus oryzae in an air-lift bioreactor. Biotechnol. Prog. 14: 699-704.

Suntornsuk W and Hang YD (1994). Strain improvement of Rhizopus oryzae for production of I (+)-lactic acid and glucoamylase. Lett. Appl. Microbiol. 19: 249-252.

Yin P, Yahiro K, Ishigaki T, Park Y, Okabe M (1998). L-(+)-lactic acid production by repeated batch culture of Rhizopus oryzae in air-lift bioreactor. J. Ferment. Bioeng. 85: 96-100.

Zhang ZY, Jin B, Kelly JM (2007). Production of lactic acid from renewable materials by Rhizopus fungi. Biochem. Eng. J. 35: 251263. 
Zhou Y, Du J, Tsao GT (2000). Mycelial pellet formation by Rhizopus oryzae ATCC 20344. Appl. Biochem. Biotechnol. 84-86: 779-789.

Žnidaršič-Plazl P (2006). The influence of some engineering variables upon the morphology of Rhizopus nigricans in a stirred tank bioreactor. Chem. Biochem. Eng. 20: 275-280.
Žnidaršič P, Komel R, Pavko A (2000). Influence of some environmental factors on Rhizopus nigricans submerged growth in the form of pellets. World J. Microbiol. Biotechnol. 16: 589-593. 